\title{
Overview of the ATLAS Insertable B-Layer (IBL) Project
}

\author{
Mario P. Giordani* \\ On behalf of the ATLAS Collaboration \\ Università degli Studi di Udine \& INFN Sezione di Trieste \\ E-mail: mario.giordani@uniud.it
}

The upgrades for the ATLAS Pixel Detector will be staged in preparation for high luminosity LHC. The first upgrade for the Pixel Detector will be the construction of a new pixel layer which will be installed during the first shutdown of the LHC machine, foreseen in 2013-14. The new detector, called the Insertable B-layer (IBL), will be installed between the existing Pixel Detector and a new, smaller radius beam-pipe at a radius of $3.3 \mathrm{~cm}$. The IBL will require the development of several new technologies to cope with increased radiation and pixel occupancy and also to improve the physics performance through reduction of the pixel size and a more stringent material budget. Two different and promising silicon sensor technologies, planar $n-i n-n$ and 3D, are currently under investigation for the IBL. An overview of the IBL project, of the module design and their qualification with particular emphasis on irradiation tests will be presented.

36th International Conference on High Energy Physics,

July 4-11, 2012

Melbourne, Australia

${ }^{*}$ Speaker. 


\section{Introduction}

ATLAS is a general-purpose detector [1] operating on the $27 \mathrm{~km}$ Large Hadron Collider (LHC) accelerator at CERN; after a two-year successful commissioning and operation up to $\sqrt{s}=8 \mathrm{TeV}$ and $\mathscr{L}=7 \times 10^{33} \mathrm{~cm}^{-2} \mathrm{~s}^{-1}$, at the beginning of 2013 the detector will enter the first of three long shutdown phases planned for the next decade in order to extend the LHC physics programme. During this period, expected to last for 20 months until the end of 2014, a fourh pixel layer named Insertable B-Layer (IBL) will be added to the present Pixel Detector [2] between a new, narrower Beryllium beam-pipe and the current inner pixel layer (B-Layer) [3]. A possible replacement of the pixel services (new Service Quarter Panel, or nSQP) and the addition of new beam monitors relying on diamond pixel sensors (Diamond Beam Monitor, or DBM) are still under discussion.

\section{Motivations}

The driving motivation of the IBL is the consolidation - and possibly the enhancement - of the tracking performance of the Inner Detector (ID) in particular in high luminosity scenarios. An additional layer of highly segmented pixel sensors helps compensating the inefficiencies caused by irreparable failures of the modules of the Pixel Detector and reduces the occurrence of ghost tracks induced by high pile-up backgrounds. Furthermore, the reduced distance of the IBL from the beam axis increases the precision on the impact parameter of tracks and, therefore, enhances the vertex recontruction and b-tagging performance of the tracking system. Despite being initially designed to operate with a luminosity of $1 \times 10^{34} \mathrm{~cm}^{-2} \mathrm{~s}^{-1}$, the Pixel Detector is expected to be still operational with an instantaneous luminosity that will exceed by at least a factor two that value: based on the current knowledge of the detector, readout saturation effects induced by the higher occupancy will be responsible of a $5 \%$ inefficiency already for $\mathscr{L}=2.2 \times 10^{34} \mathrm{~cm}^{-2} \mathrm{~s}^{-1}$.

\section{Requirements}

As anticipated, the IBL - together with the rest of the ATLAS tracker - will operate with an instantaneous luminosity up to $2-3 \times 10^{34} \mathrm{~cm}^{-2} \mathrm{~s}^{-1}$. During its operative life the detector will need to withstand - with a minimum efficiency of $97 \%$ - a Non Ionizing Energy Loss (NIEL) of $5 \times 10^{15}(1 \mathrm{MeV}) \mathrm{n}_{e q} \cdot \mathrm{cm}^{-2}$ and a total dose of $2.5 \mathrm{MGy}$, corresponding to an integrated luminosity of $550 \mathrm{fb}^{-1}$. For comparison, these values are five times more stringent than the requirements imposed for the construction of the current B-Layer.

Additional constraints affect the material budget, required not to exceed $0.015 X_{0}$ for particles at normal incidence, the power dissipation, limited to $200 \mathrm{~mW} \cdot \mathrm{cm}^{-2}$ in order to avoid thermal runaway, and the leakage current, which is required to be smaller than $100 \mathrm{pA} / \mathrm{pixel}$.

A maximum operational voltage of $1000 \mathrm{~V}$ has furthermore been foreseen in order to ensure suitable electrical insulation.

\section{Layout}

The IBL layout is shown in figure 1: it consists of a single layer of pixel sensors pre-assembled to their read-out chips (modules) and arranged in 14 staves which will be mounted on support rings 


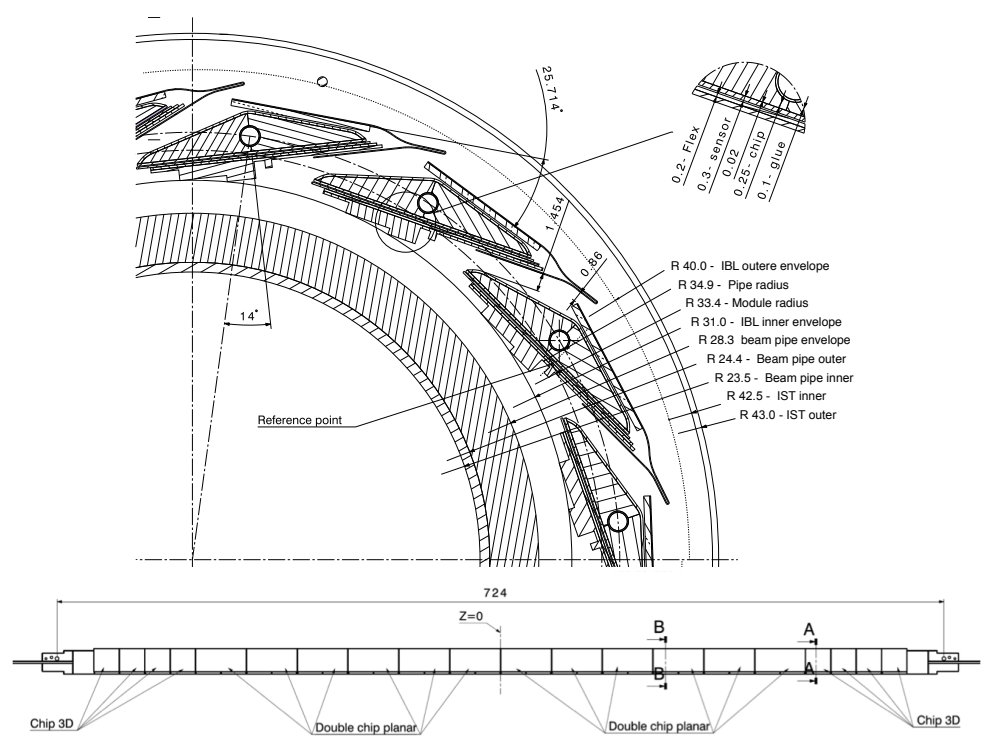

Figure 1: IBL cross-sectional view (top) and stave layout in the mixed sensor scenario (bottom) [3].

directly fastened onto a narrower $25 \mathrm{~mm}$-radius Beryllium beam-pipe; the classic turbine design is the result of tilting the staves in order to ensure complete azimuthal coverage for high $p_{\mathrm{T}}$ tracks. In this arrangement the average distance of the innermost pixel sensors from the beam-axis will be reduced from $5.05 \mathrm{~cm}$ of the current B-Layer down to $3.27 \mathrm{~cm}$. According to the current baseline assembling plan - referred to as 'mixed sensor scenario' - each stave will host 20 sensors aligned along the beam axis (z-direction): 12 two-chip planar sensors covering the central part and eight single-chip 3D sensors dedicated to the high- $\eta$ regions ${ }^{1}$, extending to $|\eta|=2.58$, where the 3D electrode geometry grants a better tracking $z$-resolution after heavy irradiation.

Limited IBL radial clearance prevents sensor shingling along a stave; for this reason, in order to maximize the $z$-coverage, thin-edge sensors have been engineered for the construction of the detector. A constant air gap of $205 \mu \mathrm{m}$ is anyway allowed between contiguous sensor-chip modules in order to provide an adequate electrical insulation.

Staves are carbon foam structures glued to v-shaped carbon fiber laminates; the carbon foam provides efficient thermal coupling to the titanium cooling pipes that are embedded within the staves. Cooling is obtained by a $\mathrm{CO}_{2}$ evaporative system that is capable of maintaining the silicon sensors at an operative temperature of $-15 \mathrm{C}^{\circ}$. Services (power, data acquisition and configuration) are routed by a multi-layer bus (flex) glued on the back of the staved from which signals are distributed to modules by means of dedicated wings.

\section{Sensors and Front-End Chip}

Two sensor technologies are involved in the IBL construction: a modern revision of the planar sensor technology, the proven reliability of which will be called to work in unprecedented operational conditions, and the 3D technology, here facing not only its first application in high energy

\footnotetext{
${ }^{1} \eta=-\ln \tan \frac{\theta}{2}$, where $\theta$ is the polar angle measured from the beam $(z)$ axis.
} 

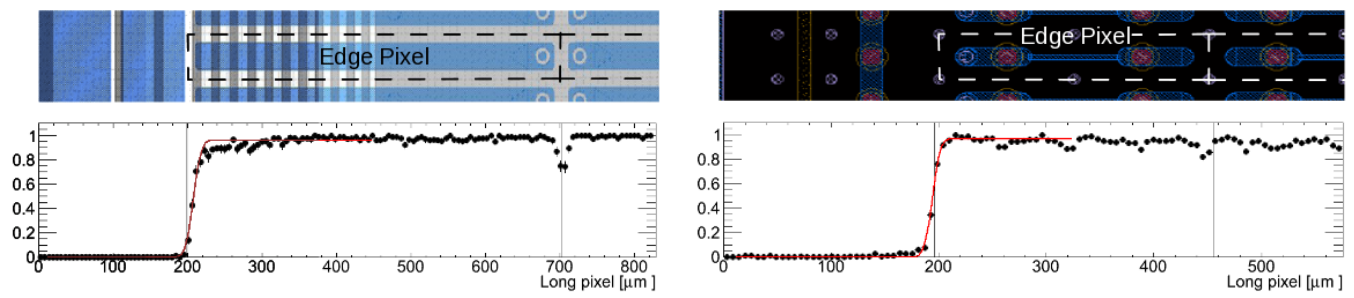

Figure 2: Thin-edge implementation (top) and overall hit efficiency (bottom) in PPS (left) and 3D-CNM (right) sensor prototypes with an applied bias voltage of $1000 \mathrm{~V}$ and $140 \mathrm{~V}$ respectively [6].

physics, but also its first-ever mass production process. Despite of being completely different, the two sensor implementations share a common footprint in order to be interfaced to the same readout chip, with two-chip planar sensor tiles having an equal longitudinal (i.e. $z$ ) dimension of two singlechip 3D sensors in order to fit a common mechanical layout; furthermore, both are characterized by the same granularity, with $250 \times 50 \mu \mathrm{m}^{2}$ pixels organized in $80 \times 336$ arrays (to be compared with the current configuration of $400 \times 50 \mu \mathrm{m}^{2}$ pixels arranged in a $18 \times 160$ matrix).

Planar pixel sensors (PPS) [4] are fabricated by $\mathrm{CiS}^{2}$ with a n-in-n design on a $200 \mu \mathrm{m}$-thick substrate, sizeably thinner than the devices used in the current Pixel Detector $(256 \mu \mathrm{m})$; the $\mathrm{n}^{+}$ pixels are isolated by means of a p-spray. Slim inactive edges ( $200 \mu \mathrm{m}$ wide) are achieved by shifting the guard rings on the p-side underneath the pixel implantations, as shown in figure 2. PPS are produced in two-chip tiles of an overall dimension of $18.59 \times 41.32 \mathrm{~mm}^{2}$; the operational voltage is expected to evolve during the device lifetime from 60 to $1000 \mathrm{~V}$.

3D pixel sensors [5] rely on a $230 \mu \mathrm{m}$-thick p-type substrate and are fabricated by $\mathrm{CNM}^{3}$ and FBK $^{4}$ with a process in which the $10 \mu$ m-radius columnar electrodes are implanted by doublesided Deep Reactive Ion Etching (DRIE) - i.e. n-type and p-type columns penetrate the substrate from opposite sides. In CNM devices columns reach a depth of about $210 \mu \mathrm{m}$ and are isolated by p-stop implantations and $\mathrm{p}$-spray on the $\mathrm{n}^{+}$and $\mathrm{p}^{+}$sides respectively. FBK devices, on the other hand, are characterized by passing-through electrodes and p-spray isolation on both sides. In both implementations, the pixel layout consists of two n-type readout electrodes connected at the wafer surface being surrounded by six p-type ohmic electrodes which are shared with the neighbouring cells. Edge isolation is achieved by a fence of ohmic electrodes which corresponds to an inactive area $200 \mu \mathrm{m}$ deep, as shown in figure 2. 3D sensors are produced in a single-chip configuration of $18.75 \times 20.45 \mathrm{~mm}^{2}$; the operational voltage is expected to range within $25-180 \mathrm{~V}$ during the device lifetime.

Due to more stringent radiation and occupancy requirements, the read-out electronics of the IBL has been completely redesigned with respect to the FE-I3, the integrated circuit currently in use in the Pixel Detector. The new front-end chip, named FE-I4 and manufactured by IBM according to a $130 \mathrm{~nm}$ CMOS technology [7], features a large footprint $\left(20.2 \times 18.8 \mathrm{~mm}^{2}\right)$ and 26880 read-out channels replicating the pixel pattern (i.e. a $80 \times 336$ array of $250 \times 50 \mu \mathrm{m}^{2}$ elements); each cell

\footnotetext{
${ }^{2}$ CiS Forschungsinstitut für Mikrosensorik und Photovoltaik GmbH, Erfurt (Germany) - www . cismst . org .

${ }^{3}$ Centro Nacional de Microelectronica, Barcelona (Spain) - www . imb-cnm. csic.es .

${ }^{4}$ Fondazione Bruno Kessler, Povo di Trento (Italy) - www . fbo . eu .
} 
comprises an independent amplifier with adjustable shaping and discriminator with independently adjustable threshold. The collected charge amplitude, measured as Time over Threshold (ToT) in units of $25 \mathrm{~ns}$ LHC bunch crossing period with a 4-bit resolution, is read at the rate of an external clock, nominally set at $40 \mathrm{MHz}$. FE-I4 chips, which are slimmed to $150 \mu \mathrm{m}$ in order to reduce the material budget, are connected to IBL sensors by bump-bonding. The FE-I4 differs from its predecessor not only in physical size but also for its logic: instead of moving all hits collected in two contiguous columns in a common end-of-column buffer as in FE-I3, each double-column is furtherly subdivided in $2 \times 2$ pixel units where hits are stored locally, with clock and trigger signals being distributed chip-wise. This results is an enhanced capability to cope with high hit rates and the overall inefficiency expected at $\mathscr{L}=3 \times 10^{34} \mathrm{~cm}^{-2} \cdot \mathrm{s}^{-1}$ reaches a level of $0.6 \%$.

\subsection{Test-Beam Results}

The performances of virgin and irradiated ${ }^{5}$ PPS and 3D sensors have been extensively studied in tests involving beams of high-energy particles, either 120 and $180 \mathrm{GeV}$ pions at CERN or $4 \mathrm{GeV}$ electrons at DESY [6]. The trajectories of the incident particles were reconstructed by means of the six-plane high-resolution EUDET telescope [8]; the effects of the magnetic field on sensors behaviour were taken into account by collecting part of the measurements within the 1.6T Morpurgo dipole magnet [9]. During data-taking, sensors were cooled and their temperature estimated at about the nominal value of $-15 \mathrm{C}^{\circ}$; the performance of the devices under test (DUTs) has been evaluated under different beam incident angles in order to recreate the situation that is expected in the actual IBL operation and study the response of high- $\eta$ modules.

Several performance parameters have been studied; the behaviour of two of them, namely hit efficiency and ToT, are depicted in figures 3 and 4 as an example. Hit efficiency is determined by extrapolating incident tracks on the DUTs surface and searching for a valid hit in the $3 \times 3$ pixel neighbourhood. As shown in figure 3, average overall values beyond $97 \%$ are typically observed; localized efficiency losses are observed in correspondence of bias grids and dots in irradiated PPS and of columnar electrodes in 3D devices when traversed by tracks at normal incidence (no effect is observed when sensors are tilted with respect to the beam direction). The different response of CNM and FBK devices visible in figure is explainable in terms of electrode geometry: in case of non passing-through electrodes (CNM), the residual bulk depth partly compensates the inefficiency.

\section{Integration and Installation}

At the time of this writing all sensors (including spares) and $70 \%$ of the read-out chips have been produced; bump-bonding of the existing components is underway at $\mathrm{IZM}^{6}$. The first stave prototype, with all modules loaded, is currently under electrical and mechanical scrutiny; all staves are expected to be assembled by the end of May, 2013, with the whole detector to be completed one month later.

The IBL installation has to be completed by the end of 2013 to fit the LHC schedule; in the original plan, the installation of the IBL is scheduled to take place in the ATLAS cavern, with

\footnotetext{
${ }^{5}$ Irradiations were performed with either neutrons or protons at different facilities and with fluences of the order of $4-6 \times 10^{15}(1 \mathrm{MeV}) \mathrm{n}_{e q} \cdot \mathrm{cm}^{-2}$.

${ }^{6}$ Fraunhofer IZM, Berlin (Germany) - www. izm. fraunhofer. de.
} 


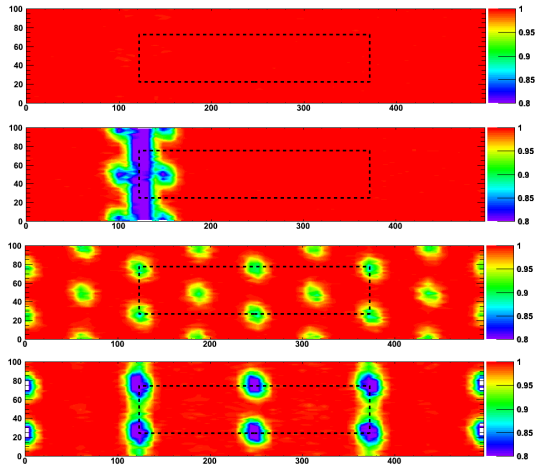

Figure 3: Hit efficiency maps for different sensor assemblies, beam at normal incidence and no magnetic field. From top to bottom: virgin and n-irradiated PPS biased at $150 \mathrm{~V}$ and $1000 \mathrm{~V}$, unirradiated 3DFBK sensor operated at $20 \mathrm{~V}$ and n-irradiated 3DCNM device biased at $160 \mathrm{~V}[6]$.
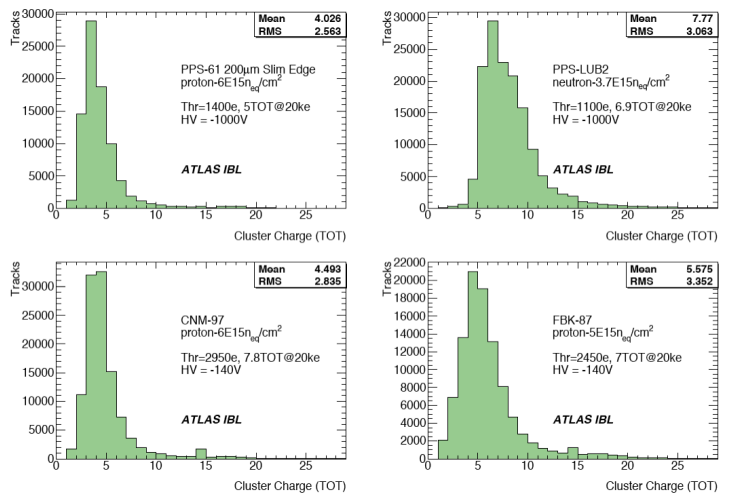

Figure 4: Raw Time-over-Threshold (ToT) spectra (in units of $25 \mathrm{~ns}$ ) for different sensor assemblies, beam at $15^{\circ}$ incidence and no magnetic field [6]. Different distributions are not directly comparable due to the unavailability of a ToT-to-charge calibration at the time of this writing.

the detector being slipped inside the current B-Layer together with the new beam-pipe after the extraction of the old one. An alternative plan foresees an installation in surface after the extraction of the whole Pixel Detector package: this eventuality would allow the recovery of some faulty pixel modules, the replacement of the pixel services with the nSQP and the installation of the DBM.

\section{Conclusions}

The ATLAS Pixel Detector will be upgraded with the insertion of a fourth pixel layer; the upgrade will occur during the next shutdown, in 2013-14. The IBL will be directly fastened on a thinner beam-pipe: as a result, the detector innermost active modules will be closer to the interaction point and will face unprecedented levels of occupancy and radiation. Planar and 3D pixel technologies have been developed and will coexist in the IBL. While pre-production prototypes are being assembled and tested, two installation procedures are still under evaluation.

\section{References}

[1] ATLAS Collaboration, JINST 3 S08003 (2008)

[2] G. Aad et al., JINST 3 P07007 (2008)

[3] ATLAS Collaboration, ATLAS TDR 19 (2010) and ATLAS TDR 19 ADD 1 (2012)

[4] M. S. Alam et al., Nucl. Instrum. Meth. A 456, 217 (2001)

[5] S. Parker, C. Kenney and J. Segal, Nucl. Instrum. Meth. A 395, 328 (1997)

[6] The ATLAS IBL Collaboration, arXiv:1209.1906 (submitted to JINST)

[7] M. Garcia-Sciveres et al., Nucl. Instrum. Meth. A 636, S155-S159 (2011)

[8] EUDET-JRA1 Collaboration, Nucl. Instrum. Meth. A 623, 399401 (2010)

[9] M. Morpurgo, Cryogenics July 1979, 411 (1979) 